\title{
Método didáctico para promover la calidad educativa en el aprendizaje de las ciencias naturales
}

\author{
DOI: https://doi.org/10.33262/ap.v3i3.66
}

\begin{abstract}
(c) (1) (8)(2)
Didactic method to promote educational quality in the learning of natural sciences
\end{abstract}

Julio César Freire Pazmiño. ${ }^{1}$

\begin{abstract}
.
Education in Ecuador In recent times it has faced changes and innovations framed in the teaching model and educational quality that aim to achieve equality and social inclusion. Objective. Didactic method to promote educational quality in the learning of natural sciences. Methodology. The quantitative and qualitative approach is used, in addition a documentary description is used that leads to the construction of knowledge framing the collection of information in the descriptive type that proposes to detail transcendental aspects related to the variables of analysis. The participants were a total of 53 eighth and ninth grade boys and girls, a questionnaire disseminated by (Chiva \& Ramos, 2015) is handled, consisting of 20 items with four choice alternatives passed on the Likert scale while the instrument was validated by the SPS Statistical Program through Cronbach's Alpha obtaining the value 0.91 excellent indicator. Results. In the planning dimension, teachers do not plan (57\%), students do not understand the content (64\%), low use of technology (64\%), restricted teamwork (68\%), insecurity and mistrust (72\%) ) insufficient motivation (68\%); in curricular subjects, reduced application of video projectors slides $(74 \%)$, carelessness in the elaboration of manipulative material $(57 \%)$, oral evaluation $(66 \%)$ inexistence of feedback $(68 \%)$ Conclusion. In the educational

\footnotetext{
${ }^{1}$ Universidad Tecnológica Indoamérica, Facultad de Ciencias Humanas de la Educación y Desarrollo Social. Carrera Educación Básica y Educación Inicial, Ambato, Ecuador, email juliofreire@uti.edu.ec. Orcid https://orcid.org/0000-0001-9936-5605
} 
institution, educators still use traditional methods that increase the transmission of content from the discipline, dependence and memorization of essences that affect didactics, autonomy and problem solving. On the other hand, there is a need to use computer programs where the student has an active role based on the connections of ideas, innovative practice and academic reinforcement.

Keywords: learning, educational quality, Natural Sciences, didactic method.

\section{Resumen.}

La educación en el Ecuador Durante los últimos tiempos ha enfrentado cambios e innovaciones enmarcadas en el modelo de enseñanza y la calidad educativa que tienen como finalidad alcanzar la igualdad y la inclusión social. Objetivo. Método didáctico para promover la calidad educativa en el aprendizaje de las ciencias naturales. Metodología. Se emplea el enfoque cuantitativo y cualitativo, además se utiliza una descripción documental que conduce a la construcción del conocimiento enmarcando la recolección de información en el tipo descriptivo que propone detallar aspectos trascendentales relacionados con las variables de análisis. Los participantes fueron un total de 53 niños y niñas de octavo y noveno grado, se maneja un cuestionario difundido por (Chiva \& Ramos, 2015), conformado por 20 ítems con cuatro alternativas de elección pasados en la escala de Likert mientras el instrumento fue validado por el Programa Estadístico SPS a través del Alfa de Cronbach obteniendo el valor 0,91 indicador excelente. Resultados. En la dimensión de planificación los maestros no realizan planificaciones (57\%), los educandos no comprenden el contenido (64\%), escasa utilización de tecnología (64\%), restringido trabajo en equipo (68\%), inseguridad y desconfianza (72\%) insuficiente motivación (68\%); en materias curriculares, reducida aplicación de videos proyectores diapositivas (74\%), descuido en la elaboración de material manipulativo (57\%), evaluación oral $(66 \%)$ inexistencia de retroalimentación $(68 \%)$ Conclusión. En la institución educativa los educadores aún permanecen utilizando métodos tradicionales que incrementan la transmisión de contenidos desde la disciplina, la dependencia y memorización esencias que aquejan a la didáctica, autonomía y solución de problemas. Por otra parte existe la necesidad de emplear programas informáticos donde el estudiante tenga un papel activo en base a las conexiones de ideas, la práctica innovadora y el refuerzo académico.

Palabras clave: aprendizaje, calidad educativa, Ciencias Naturales, método didáctico.

\section{Introducción.}

El tema de estudio, responde a la necesidad de mejorar la calidad educativa en los sistemas institucionales, pretende puntualizar los diferentes conceptos y realidades de la práctica docente enfocado en el método didáctico para promover el aprendizaje significativo en las asignaturas, de forma especial en ciencias naturales. 
Los centros educativos y las aulas se constituyen en espacios que dependen de las decisiones políticas y técnicas que adopte el sector gubernamental para mejorar la educación, caso contrario se continuará trabajando en el mismo currículo sin avanzar hacia el progreso; se profundiza en el estudio de los modelos tradicionales como factor que dificulta la comprensión del conocimiento y la enseñanza de contenidos conceptuales, por lo expuesto, con el propósito de responder a las necesidades de cada estudiante en función de sus características personales e individuales, se considera trascendental aplicar estrategias que respondan al modelo actual y beneficien en la adquisición de competencias que facilita la innovación en forma de enseñar, la asimilación, las actitudes junto con el pensamiento crítico y lógico.

En el país ecuatoriano, el régimen educativo, se sustenta en leyes, normativas y reglamentos que conducen hacia una instrucción eficiente y productiva; desde una perspectiva holística la Constitución de la República del Ecuador (2015) que reconoce al estudiante como de los procesos educativos; Sin embargo, según (González, 2017 ) en varias instituciones educativas la ausencia de los maestros ha perjudicado en el rendimiento académico al interrumpir la continuidad de los contenidos determinados para el área generando un retraso en el cumplimiento del programa formativo; el escaso reconocimiento al esfuerzo y perseverancia desmotiva en la adquisición de hábitos de estudio; el insuficiente acompañamiento del padre y la madre repercute en la toma de decisiones y resolución de problemas con autonomía y confianza.

Según (Herrera, Montes, Tapia, \& Barros, 2017) manifiestan: en busca de impulsar el desempeño académico de los estudiantes en la provincia de Tungurahua diferentes instituciones educativas intentan conocer los problemas que enfrentan los educandos al igual que sus expectativas con el propósito de efectuar acciones en apoyo que favorezcan en la capacidad intelectual y eviten su deserción; (Villavicencio, 2018) detecta que el principal problema es el insuficiente acceso a las tecnologías de la información y comunicación que perjudica en el rendimiento, aspecto que conlleva al fracaso escolar; incluye que la falta de aplicación de estrategias incrementa un deterioro en el aprendizaje.

Por lo expuesto se considera trascendental efectuar un estudio enfocado en la identificación de los factores que perjudican en la adaptación de métodos didácticos para promover la calidad educativa en el aprendizaje de las diferentes asignaturas especialmente de ciencias naturales. Los investigadores (Hernández \& Guárate, 2017) en la investigación vinculada con la metodología pedagógica y didáctica afirma que la escasa utilización de materiales educativos perjudica en la captación del contenido, el desarrollo de las capacidades interpretativas analíticas y de síntesis; coadyuvando en la repetición memorística, lo que limita la consolidación de temáticas desde la disciplina científica.

El presente estudio propone analizar el método didáctico utilizado por los maestros en el aprendizaje de las ciencias naturales, el trabajo de campo se efectuará con los estudiantes de Básica Superior (Sub-nivel 4), correspondiente a $8^{\circ}$ y $9^{\circ}$ grados de Educación General Básica de la Unidad Educativa Intercultural Bilingüe Tamboloma; con base en los resultados obtenidos en la aplicación de la encuesta y a través del cuestionario 
estructurado se recabará información trascendental que beneficiará en el establecimiento de conclusiones; por lo expuesto, en la indagación se plante el objetivo: determinar la importancia del método didáctico para promover la calidad educativa en el aprendizaje de las ciencias naturales.

\section{Impacto del método didáctico en la calidad educativa y los aprendizajes académicos}

El método didáctico se incluyen en la gestión estratégica que promueve y asegura la calidad educativa; algunos teóricos entre ellos (Quintana, 2018) afirman: en el trabajo formativo se incorpora las prácticas contemporáneas, por su insuficiente conceptualización ha perjudicado en la familiaridad en diferentes concepciones, generando limitaciones en la obtención de los saberes. Actualmente durante el progreso de la clase el empleo de métodos beneficia en la aplicación de estrategias, técnicas, actividades y tareas que favorecen en la comprensión. Según (López \& Alvarado, 2017) afirma que la pedagogía y la didáctica al formar parte de la Filosofía de la educación se constituye en un elemento indispensable para el control de proceso educativo que mediante la ciencia favorece en el desarrollo mental, la formación del carácter y los modos de aprendizaje; es decir, los métodos didácticos desde el enfoque constructivista plantean una interrelación dinámica que pretende contribuir en el mejoramiento de las destrezas con criterio de desempeño, permitiendo que el educando resuelva problemas en las situaciones que la vida le presenta.

El modelo didáctico se vincula con la metodología aplicada en las asignaturas impartidas en el ambiente escolar; (Zabalza, 2017) considerando que la utilización de diversas estrategias aporta en el trabajo en equipo, siendo necesaria la intervención del maestro para alcanzar la capacidad de análisis, la síntesis e integración de contenidos; (Ayala \& Ayala, 2018) de esta forma propicia la profundización y generalización de determinados temas encaminando hacia el logro de niveles de conocimientos y habilidades durante el proceso de enseñanza aprendizaje. Es decir contribuye en la construcción del conocimiento, teniendo como finalidad sintetizar los procesos la práctica docente mediante la reflexión, comprensión y organización de acuerdo con los propósitos definidos dentro del documento curricular.

El análisis de los métodos didácticos, según (López M. , 2015) inician con el tradicional que contribuye en la formación del carácter y la conducta de los estudiantes a través de la voluntad la virtud y rigor de la disciplina, de esta manera conlleva a la tradición en la enseñanza, al emplear la imitación, monotonía y transferencia verbal donde el maestro dicta sus clases bajo el contenido textual, siendo el estudiante únicamente en receptor pasivo. Los ejes centrales son el docente y el texto, enfatiza en el escrito y la transmisión de conocimientos y valores. Por lo expuesto se deduce que la escasa la utilización de técnicas mantiene un sistema rígido que dificulta la participación de forma dinámica y la innovación, (Necuzzi, 2018) por tanto resalta su importancia en la memorización y repetición de conceptos habituando al educando en el área pasiva al cumplir las disposiciones en un ambiente autoritario. 
En el método didáctico conductista o procesos tecnológicos, (Mayoral, 2016) la conducta es productiva, el método se enfoca en la fijación y el control de los objetivos instruccionales formulados con precisión y reforzados meticulosamente mediante instrucciones diseñadas por expertos y administrados por los maestros. Las actividades pueden presentarse en manera escrita o utilizando un software, elementos que permiten la adquisición de conocimientos experiencias y competencias que benefician al área intelectual. (Patón, Fernández, \& Ferreiro, 2018), mientras el método didáctico activista está centrado en las destrezas y parte del desarrollo de las actitudes, atiende al proceso de enseñanza y aprendizaje de forma espontánea; destaca la argumentación y libertad para mejorar las habilidades de acuerdo a los contenidos que el educando desee seleccionar; la disciplina es un obstáculo en los procedimientos educativos.

Para, (Santana \& Corral, 2020) el método didáctico alternativo, se centra en el seguimiento de la evolución del conocimiento de los educandos junto con la actuación y comportamiento de los maestros en el desarrollo del proyecto, (Ochoa, 2019) tiene como meta alcanzar un mejor nivel intelectual a través de diferentes actividades que benefician en la construcción de los saberes; inicia en la conceptualización las necesidades y condiciones de un ambiente que estimula las experiencias y facilitan al educando el acceso a las estructuras cognitivas superiores. Tomando el criterio de los autores mencionados, los métodos didácticos contribuyen en las capacidades, potencialidades e intereses del alumnado, teniendo el propósito de lograr un trabajo productivo que contribuya en la superación de las desigualdades sociales y educativas.

A modo de conclusión, se deduce que los estudiantes mediante la utilización de métodos didácticos asumen un compromiso con el aprendizaje basado en el ejercicio comunicativo, y la investigación-acción, beneficiando en la participación activa, siendo su función, contribuir en las formas de organización y la convivencia en las instituciones educativas; de esta forma se concreta las concepciones de educación, desde la misión, visión y práctica de valores institucionales orientando en la realización de actividades encauzadas al cumplimiento de los propósitos educativos.

\section{Métodos didácticos que utilizan los maestros durante el proceso de enseñanza y aprendizaje}

La visión constructivista cognitiva mantiene que el educando es creador y constructor de su propio conocimiento y destrezas mediante la utilización de métodos que incluyen las actividades y los recursos que benefician en el trabajo de la mente; beneficia en la interpretación y explicación de sus experiencias, de esta forma reestructura sus concepciones en grupos de trabajo.

Para (Iturralde, Bravo, \& Flores, 2017) los métodos didácticos despliegan esquemas que contienen, diversas acciones, técnicas y medios aplicados por los maestros, con el transcurso del tiempo han intervenido en la evolución de la ciencia, es así como el método didáctico de transmisión o recepción se centraliza en la labor del docente y en los contenidos de aprendizaje, es especial en los aspectos metodológicos y el contexto; 
generando afectaciones en el desempeño global de los niños y niñas; el conocimiento producido por la investigación científica será difundido mediante la expresión verbal y la utilización de textos vinculados con las diferentes asignaturas.

El método didáctico por descubrimiento se caracteriza porque el profesor guía el proceso de educativo mediante procedimientos y actitudes que benefician en el comportamiento y atención, componentes que conllevan el conocimiento a partir de la interacción con la realidad; en las ventajas menciona, la actitud crítica y propositiva de los estudiantes, la motivación y el aprendizaje; (Tünnermann, 2011) la aplicación de estrategias básicas favorece en la enseñanza de ciencias naturales integradas por experimentos y consultas en materiales existentes en la biblioteca de aula, es trascendental la construcción de maquetas, el diseño de álbumes, la producción y redacción de mapas conceptuales; según (Olivo, 2017) la utilización del diccionario científico junto con la realización de recorridos y visitas a los lugares donde se desarrolla la acción incluye paseos o excursiones guiados por los docentes en los alrededores de la comunidad, de esta forma se fortalece las habilidades de observación desde el aspecto crítico propositivo; por lo expuesto, se recomienda determinar la relación entre los temas aprendidos en la sala de clase y el lugar, siendo necesario la elaboración de un plan de tareas para optimizar el tiempo y los recursos.

La educación de las ciencias naturales, apoya en la valoración y toma de decisiones del maestro sobre un contenido y el énfasis que demuestra en la enseñanza; según (Orellana, Quintanilla, \& Páez, 2018) menciona el método didáctico del diccionario científico que respalda al estudiante en el entendimiento y discernimiento de significados exterioriza de forma organizada palabras conocidas durante las clases en el aula, es significativo al desarrollar habilidades, destrezas y capacidades empleando estrategias y criterios que benefician en la búsqueda selección y organización de datos. Además, incluye los métodos tecnológicos enfocados en la transmisión del conocimiento utilizando las tecnologías, (software) coopera en el proceso de aprendizaje, logrando la interacción entre los contenidos, los saberes y las experiencias. En los estudios efectuados por (Herrera \& Zapata, 2018) hace referencia al método didáctico como cápsula científica que consiste en presentar información relevante a través de un lenguaje sencillo que atrae la atención de los lectores; por lo expuesto se considera trascendental utilizar fotografías, imágenes o ilustraciones que promuevan la investigación en los escolares.

Una de las experiencias de mayor recurrencia en la enseñanza de las ciencias naturales ha sido el empleo de estrategias que relaciona los entornos educativos, (Muñoz, Montenegro, \& Aparicio, 2017) afirman que el método didáctico activista, tiene por finalidad educar a los estudiantes en la realidad, se sustenta en los intereses de los educandos en el trabajo individual, la manipulación, la experimentación, la invención, el descubrimiento; el educador es responsable de elaborar recursos concretos, permitiendo al individuo pensar y actuar a su manera a través de la experiencia. Mientras en la educacion pre escolar menciona que el método didáctico Montessori, que emplea actividades motrices y sensoriales, aporta en el desarrollo integral de los niños y niñas; entre sus objetivos esta descubrir y ayudar en el alcance del potencial como ser humano, 
mediante los sentidos, en un contexto preparado y aplicando la observación científica de un profesor entrenado en el uso de materiales especializados que benefician en la adquisición de potencialidades que favorecen en la toma de decisiones en los diferentes contextos.

En síntesis, los métodos didácticos en Ciencias Naturales, permiten la simbolización y representación de la tarea de enseñanza y aprendizaje, requiriendo el desarrollo de prácticas áulicas y el conocimiento de las relaciones existentes partiendo de los aspectos psicológicos, epistemológicos, principios y procesos de acción, que engloba las concepciones de ciencia, las estrategias, los recursos y clima en el aula componentes que conllevan al pensamiento crítico, creativo, argumentativo.

\section{Causas que afectan en la aplicación de métodos didácticos en el aprendizaje de las ciencias naturales.}

Al hablar de métodos didácticos se hace referencia a las estrategias que utiliza el educador en el aprendizaje y la habilidad de los educandos para aprender; en la exploración efectuada por (Villegas, Muñoz, \& Villegas, 2009) entre las causas que afectan en la utilización de modelos didácticos las siguientes: desconocimiento del docente en su manejo y aplicación, insuficiente capacitación e innovación en el uso de metodologías innovadoras, desinterés del profesorado en el empleo de procesos dinámicos e interactivos; (Pérez, 2016) varios se concentran en el desarrollo individual; unos cuantos se enfocan en las ventajas del trabajo en grupo, mientras que otros pretenden desarrollar hábitos de investigación. Investigadores como (Ortega \& Puente, 2019) afirman que algunos maestros no describen modelos sino formas elementales de enseñar; argumentar, narrar, describir, observar, analizar, construir, estudiar y repetir.

Continuando con los juicios valorativos anteriores, (Ortega \& Gil, 2018) expone que el insuficiente interés en el empleo de métodos didácticos de forma paulatina ha influido negativamente en la curiosidad, investigación y la motivación por el aprendizaje de los contenidos tratados en el aula, introduciendo un proceso pasivo donde prevalece la desmotivación y el fracaso académico. (García \& Buitrago, 2017), el enfoque teórico desmotiva a los estudiantes en la práctica de hábitos de estudio; el desinterés genera dificultades en la comprensión; la ciencia es considerada una materia enciclopédica, sobresale la memorización y retención. Por lo expuesto, en la mayoría de instituciones educativas la utilización desmedida de los libros de texto, las inadecuadas programaciones, el aislamiento de los temas científicos, la evaluación cuantitativa y exámenes dirigidos a calificar el aspecto memorístico, son causas que repercuten en la adquisición del saber.

\section{Metodologia}

Según Aguilar (2016) el enfoque cuantitativo se caracteriza por ser secuencial y probatorio, emplea la experimentación, el análisis y determinación de conclusiones. Por tanto se efectúa la recolección con base en una medición a través de procedimientos e instrumentos estandarizados el mismo que es aceptado en la comunidad científica; así, 
favorece en la recopilación de los datos (cantidades) que son analizados con métodos estadísticos.

Para Sampieri, Fernández, \& Baptista (2014) el enfoque cualitativo se sustenta en la indagación y exploración basado en la observación, se centra en la comprensión, cumple con postulados constructivistas al construir la interpretación enfocándose en la transformación de la realidad observable. Es decir permitió estudiar el aspecto real en su contenido natural, con una visión empírica mediante la producción de datos descriptivos, donde el investigador desarrolla conceptos y teorías preconcebidas siguiendo un diseño flexible.

La revisión documental por medio del procedimiento científico y a través de un proceso sistemático, facilitó la indagación, recolección, organización, análisis e interpretación de los datos en referencia al modelo didáctico y la calidad educativa en el aprendizaje de las ciencias naturales; además, condujo a la construcción del conocimiento, sobresale en la selección documentos de contenidos relevantes (disposición científica, autores y año de realización); información obtenida del Google y Google académico, estudios de universidades, libros digitales e impresos.

La indagación, se enmarcó en el estudio descriptivo; Burgo, León, Cáceres, Pérez, \& Espinoza, (2019) menciona, "que proponen detallar las propiedades, las características y los perfiles importantes de personas, grupos, comunidades que se sometan a un análisis; facilitó la medición de la información recolectada para posteriormente efectuar una descripción, e interpretación sistemática con sustento en la realidad. Se resalta que la investigación no experimental, con diseño transversal y el tipo exploratorio promovió el establecimiento de las conclusiones.

\section{Participantes}

Los estudiantes objeto de estudio cursan el $8^{\circ}$ y $9^{\circ}$ grados de Educación General Básica de la Unidad Educativa Intercultural Bilingüe Tamboloma en una totalidad de 53 educandos, comprendidos en las edades de 12 a 15 años. Participó toda la poblacion, por esa razón no se tomó una muestra concluyente, acontinuación se detalla la población.

Tabla 1. Población y muestra

\begin{tabular}{llllll}
\hline Nivel & Nivel & \multicolumn{2}{l}{ Personas objeto de estudio. } & Subtotal & Porcentaje \\
\cline { 3 - 5 } & & Femenino & Masculino & \\
\hline Educación General & Octavo & 8 & 18 & 26 & $49 \%$ \\
Básica Superior & Noveno & 10 & 17 & 27 & $51 \%$ \\
& & & & 53 & $100 \%$ \\
& Sub Total & 18 & 35 & & \\
\hline
\end{tabular}

Fuente: Secretaría. Unidad Educativa Intercultural Bilingüe Tamboloma.

Elaboración: Investigador.

Participaron un total de 53 estudiantes correspondientes al nivel de Educacion General Básica Superior comprendidos en las edades de 12 a 15 años de edad, población de estudio que cumplió con los criterios de inclusión, es decir, firmaron la autorizacion para 
continar con la indagación; por tal motivo no se tomo una muestra determinada.

\section{Instrumentos}

El estudio presentado, incluye la adaptación de la escala para evaluar las estrategias de gestión e intervención docente en educación primaria; (Chiva \& Ramos, 2015) es decir se efectúa un acercamiento cualitativo en la valoración de contenido mediante el juicio de expertos en el área, el propósito es lograr la identificación y revisión de elementos relevantes vinculados con los modelos didácticos utilizados por los docentes para promover la calidad educativa en el aprendizaje de las ciencias naturales.

En cuando a la aproximación cuantitativa, se utiliza el Programa Estadístico SPSS, para efectuar la validación de instrumentos que serán aplicados en la recolección de información numérica, obteniendo datos a partir de la escala de Likert; la selección de ítems y el análisis de consistencia interna.

\section{Diseño de la Escala (Validez de contenido y de constructo).}

Para efectuar la recolección de información, se realiza una revisión bibliográfica en un proceso sistemático y riguroso de investigaciones previas vinculadas con la variable modelo didáctico utilizado por el docente y la calidad educativa; acciones que fueron efectuadas mediante documentos que incluyen: Nomenclatura de artículos de revistas sobre ciencias sociales y humanidades; Bases de Datos de Información del Ministerio de Educación y Ciencia; entre otras.

A partir de las definiciones extraídas se define que el modelo didáctico de enseñanza debe dar respuesta a las interrogantes: ¿cómo enseñar? y ¿cómo ayudar a aprender?, de forma concreta se pretende aportar en la gestión e intervención docente (o pedagógica), en las diferentes formas de enseñar en el aula, para facilitar o guiar el aprendizaje de los educandos.

Una vez determinado el constructo metodología aplicada por el profesorado se realiza el análisis de instrumentos y escalas diseñadas por otros investigadores e investigadoras, se sintetizan 4 capacidades: planificación del proceso de enseñanza y aprendizaje, tipos de actividades, materiales curriculares y evaluación (Chiva \& Ramos, 2015). Siguiendo en el orden, se extrae una totalidad de 20 ítems, procediendo al diseño de un cuestionario; el propósito fue extraer los de mayor significación relacionados con el método didáctico, atendiendo a la estimación de los métodos empleados por el profesorado; aplicando en su valoración una escala tipo Likert de 4 puntos, donde 1 equivale a nunca, 2 a veces, 3 casi siempre y 4 siempre.

Tabla 2. Encuesta aplicada a los estudiantes

\begin{tabular}{|c|c|c|c|c|c|}
\hline \multirow[t]{2}{*}{$\mathbf{N}^{\circ}$ ITEN } & \multirow{2}{*}{$\frac{\text { DIMENSIÓN }}{\text { Planificación del proceso de enseñanza y aprendizaje }}$} & \multicolumn{4}{|c|}{$\begin{array}{r}\text { ESCALA DE } \\
\text { VALORACIÓN }\end{array}$} \\
\hline & & 1 & 2 & 3 & 4 \\
\hline 1 & $\begin{array}{l}\text { El maestro o la maestra solicitan su opinión para planificar las actividades que efectuará } \\
\text { en el aula. }\end{array}$ & 11 & 12 & 20 & 10 \\
\hline 2 & El contenido es preparado por el docente antes del inicio de la clase. & 9 & 27 & 9 & 8 \\
\hline
\end{tabular}




\begin{tabular}{|c|c|c|c|c|c|}
\hline 3 & $\begin{array}{l}\text { En el ambiente educativo el educador emplea diferentes actividades para lograr la } \\
\text { comprensión de contenidos }\end{array}$ & 12 & & 12 & 9 \\
\hline 4 & En el ambiente académico se utiliza equipo tecnológico. & 8 & 26 & 9 & 10 \\
\hline 5 & $\begin{array}{l}\text { El uso de las nuevas tecnologías facilita la comprensión, atención y asimilación de } \\
\text { conceptos. }\end{array}$ & 12 & 23 & 10 & 8 \\
\hline & Tipos de actividades & 1 & 2 & 3 & 4 \\
\hline 6 & Los debates dirigidos por el maestro o maestra fortalecen el trabajo en equipos & 13 & 23 & 19 & 7 \\
\hline 7 & Las actividades que propone el maestro o la maestra en el aula despiertan su creatividad. & 9 & 25 & 11 & 8 \\
\hline 8 & En los temas o trabajos que expone en la clase demuestras seguridad y confianza. & 9 & 29 & 6 & 9 \\
\hline 9 & Le gusta realizar trabajos de investigación y descubrimiento. & 8 & 26 & 10 & 9 \\
\hline 10 & Le agrada realizar actividades fuera del ambiente educativo. & 20 & 16 & 8 & 9 \\
\hline \multicolumn{6}{|c|}{ Materiales Curriculares } \\
\hline 11 & $\begin{array}{l}\text { Es habitual que en clase el maestro o la maestra utilice medios audiovisuales (vídeo, } \\
\text { proyector de diapositivas,...). }\end{array}$ & 19 & 20 & 6 & 8 \\
\hline 12 & $\begin{array}{l}\text { Es habitual que en clase se use materiales elaborados por el propio maestro o la maestra } \\
\text { (fichas, esquemas, apuntes...). }\end{array}$ & 15 & 24 & 9 & 5 \\
\hline 13 & $\begin{array}{l}\text { Es habitual que en clase se utilicen materiales elaborados en equipo (elaborados por } \\
\text { el profesorado). }\end{array}$ & 10 & 20 & 12 & 11 \\
\hline 14 & $\begin{array}{l}\text { Es habitual que en clase se utilicen materiales impresos editados (libros de texto, } \\
\text { mapas ............... ). }\end{array}$ & 6 & 12 & 20 & 15 \\
\hline 15 & $\begin{array}{l}\text { Es habitual que en clase se utilicen material manipulativo (de laboratorio, juegos, } \\
\text { instrumentos...). }\end{array}$ & 14 & 29 & 7 & 3 \\
\hline \multicolumn{6}{|c|}{ Evaluación } \\
\hline 16 & El docente evalúa a través de controles orales después de cada tema o lección. & 5 & 13 & 28 & 7 \\
\hline 17 & El educador o educadora realiza un seguimiento personal de cada estudiante. & 5 & 12 & 32 & 4 \\
\hline 18 & $\begin{array}{l}\text { La evaluación final incluye la presentación de tareas, deberes, pruebas orales - escritas } \\
\text { y exámenes }\end{array}$ & 0 & 0 & 0 & 53 \\
\hline 19 & $\begin{array}{l}\text { Para obtener buenas calificaciones cumple con las actividades y trabajos con } \\
\text { responsabilidad. }\end{array}$ & 2 & 11 & 34 & 6 \\
\hline 20 & El maestro o la maestra realiza retroalimentación de contenidos & 20 & 16 & 9 & 8 \\
\hline
\end{tabular}

Fuente: Compilado por investigador. (Chiva \& Ramos, 2015).

\section{Validación del instrumento}

Tabla 3. Encuesta aplicada a los estudiantes

\begin{tabular}{llrr}
\hline \multicolumn{5}{c}{ Resumen del procesamiento de los casos } \\
\hline & N & \% \\
\hline \multirow{3}{*}{ Casos } & Válidos & 53 & 100,0 \\
& Excluidos $^{\mathrm{a}}$ & 0 &, 0 \\
& Total & 53 & 100,0 \\
\hline
\end{tabular}

Fuente. Encuesta aplicada a los estudiantes. Resultado Programa Estadístico, SPSS

Tomando como referencia en la tabla de resumen de procesamiento de casos basada en la lista de todas las variables de procedimiento se obtiene un total de 50 casos inválidos con equivalencia al $100 \%$

Tabla 4. Estadísticos de fiabilidad

Alfa de Cronbach
Alfa de Cronbach basada en los
elementos tipificados
$\mathrm{N}$ de elementos

,959

Fuente. Encuesta aplicada a los estudiantes. Resultado Programa Estadístico, SPSS 
El instrumento en el estudio fue validado, en el Programa Estadístico SPSS, evidenciado en la tabla 2, alcanzando el valor estadístico de fiabilidad de 53 casos equivalente al 100\%, un Alfa de Cronbach basada en elementos tipificados del 0.961. Según Villanueva,(2016), el valor de fiabilidad en el estudio que incluye una investigación básica oscila entre 0.7 y 0.8; por tal razón equivale al criterio de excelente (Ortiz, 2021).

\section{Análisis estadístico}

Gráfico 1. Análisis y porcentajes

\section{Título del gráfico}

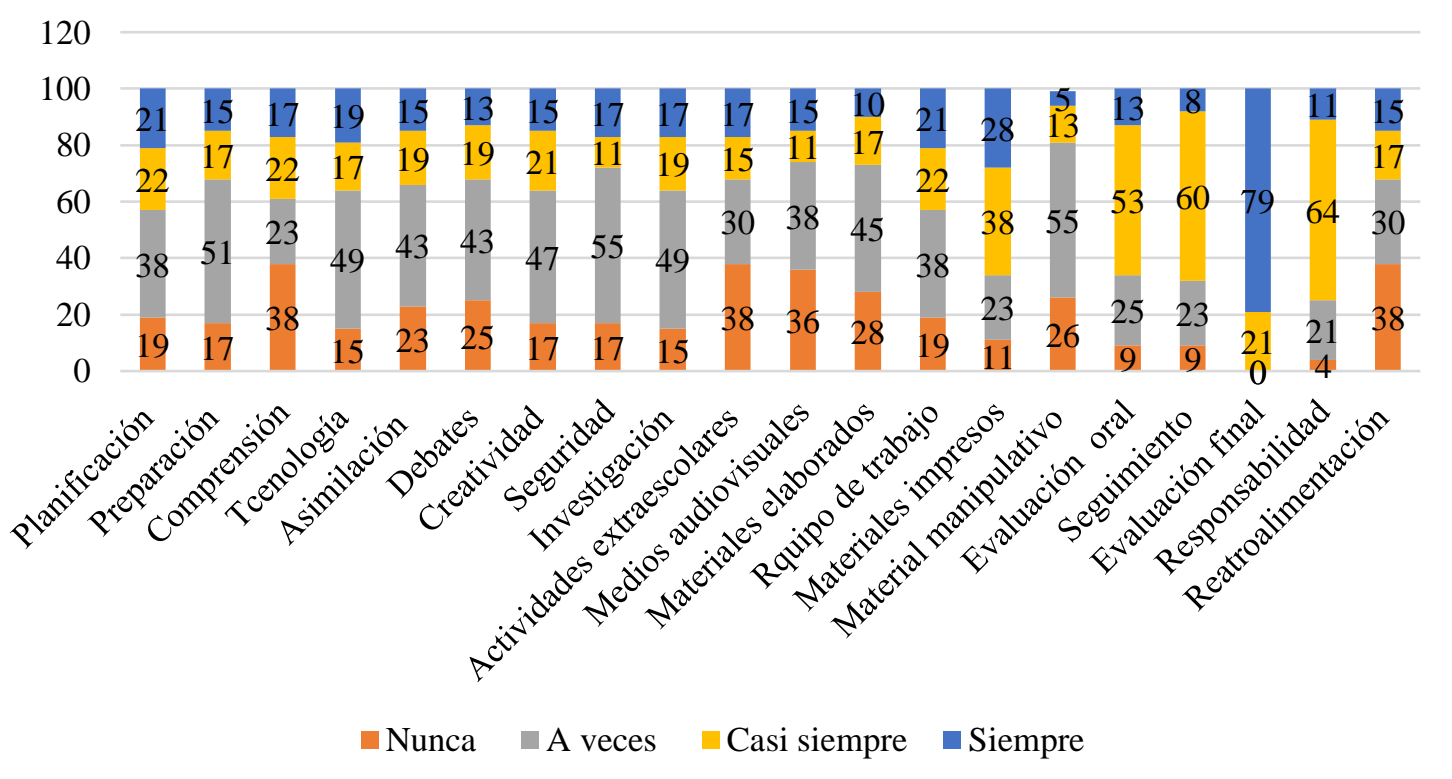

Fuente. Encuesta aplicada a los estudiantes. Resultado Microsoft Excel

De una totalidad de 53 niños y niñas equivalente al 100\%, en la dimensión planificación del proceso de enseñanza y aprendizaje el $68 \%$ de los maestros y las maestras no preparan el contenido antes del inicio de clases factor limitante en el desarrollo de la creatividad; desde esta perspectiva la clase improvisada puede generar una barrera en el logro de los aprendizajes de los educandos al afectar en la recolección, organización y secuencia de los resultados esperados.

En la segunda dimensión tipos de actividades el $72 \%$ de niños y niñas exterioriza inseguridad y desconfianza al momento de exponer la clase, aspecto que genera barreras en el diálogo, la resolución de conflictos y problemas, deteriorando en la motivación y la comunicación entre los actores del aprendizaje.

En la tercera dimensión denominada materiales curriculares se visualiza que él 74\% de maestros y maestras no utiliza medios audiovisuales lo que perjudica en el aprendizaje, matiz que interviene en el contexto educativo en la adquisición de definiciones, conceptos destrezas y habilidades influyendo de forma negativa en la incorporación de nuevos recursos comportamientos y prácticas de enseñanza que desde la labor pedagógica, didáctica y currículum incrementa dificultades en el entendimiento y la comprensión. 
En la cuarta dimensión vinculada con la evaluación se obtiene que en un mayor porcentaje los maestros y maestras realizan la sumatoria de todas las actividades efectuadas dentro y fuera del área escolar además el $75 \%$ exterioriza que es necesario fomentar la responsabilidad en las tareas escolares y escolares para alcanzar la calidad educativa que incluye la pedagogía, la metodología, las herramientas, los recursos que el profesorado emplea en el progreso de sus clases.

\section{Resultados.}

Después de efectuado el análisis e interpretación de datos estadísticos se obtiene los siguientes resultados:

En la identificación del método didáctico empleado por los docentes para promover la calidad educativa en el aprendizaje de las ciencias naturales se obtiene que el $57 \%$ de maestros no planifica las actividades, un 32\% demuestra desinterés en la preparación de clases antes de impartir el contenido, el $61 \%$ no usa estrategias que favorezcan en el entendimiento, el $64 \%$ no utiliza equipo tecnológico, el $66 \%$ no maneja las tecnologías de la información y comunicación; sin embargo un $43 \%$ casi siempre y a veces emplea la planificación curricular.

En el diagnóstico de la metodología aplicada por el profesorado para promover la calidad educativa en el área de ciencias naturales, se resalta que en la institución se mantiene un tipo de educación con sustento en la pedagogía tradicional; se obtiene que el 64\% aplica material receptivo y repetitivo, el $64 \%$ limita la curiosidad y la creatividad e investigación y el descubrimiento acciones que han influido en los niños y niñas; el 68\% no efectúa con responsabilidad las tareas extraescolares. A modo de síntesis (Gómez, 2012) afirma, en el sistema educativo es trascendental el uso de los métodos didácticos, debiendo ser adaptados a las características de cada estudiante. Para (Salazar, 2018) los procesos y procedimientos benefician en la capacidad del estudiantado, al establecer una relación entre los aspectos teóricos y sus aplicaciones prácticas; en concordancia con los criterios (Gómez, 2016) manifiesta que existe a necesidad de fortificar los saberes mediante estrategias que ayudan en el desarrollo de contenidos conceptuales, los educandos consiguen habilidades que facilitan la discusión, el análisis consensuado, la asimilación del nuevo contenido. Por lo expuesto, es significativo que el maestro incluya en la planificación metodologías que propician una enseñanza intencional, reflexiva, consciente y autorregulada.

En cuanto a materiales curriculares el $73 \%$ imparte los contenidos a través de clases magistrales, sobresale la utilización de libros, enciclopedias o impresos escritos de esta forma el $81 \%$ no aplica material de laboratorio juegos o instrumentos manuales, como manifiesta (Pazmiño, Núñez, López, \& Pazmiño, 2019) en las instituciones educativas es importante que los maestros y maestras empleen estrategias innovadoras que favorezcan en el trabajo en equipo, la cooperación y refuerzo académico aplicando los principios básicos del método científico. 


\section{Conclusiones.}

- En el presente estudio se deduce que es relevante la incorporación de estrategias y métodos innovadores que beneficien en los procesos de enseñanza aprendizaje, siendo así la tecnología un componente esencial para disminuir los metodologías tradicionales logrando en el ambiente educativo la motivación, interacción y el trabajo en equipo, elementos que benefician en la labor pedagógica brindando una formación práctica y funcional.

- Es trascendental entender que el proceso de enseñanza aprendizaje debe ser innovador por tal razón la utilización de métodos de nuevas estrategias didácticas tecnológicas promueven el cambio en la práctica del maestro; de forma usual se asocia el manejo de las tecnologías con los objetivos propuestos en el plan curricular y el desarrollo de competencias que conllevan a la investigación y el descubrimiento, elementos que benefician en la creatividad, las experiencias, la capacidad de construir, el conocimiento, la participación activa, la disposición del estudiante para aprender desde la interacción con los medios informáticos.

- Existe la necesidad de mejorar la planificación de clase en el aspecto técnico y dinámico con un enfoque constructivista, donde los educandos a través de la organización de la información desarrolla los conocimientos, adquiere destrezas y habilidades; mejora los procesos y procedimientos, responde a propósitos claros actitudinales y comportamentales particular que orienta a la selección y sistematización de la teoría y la práctica; en este sentido beneficia en el lenguaje y los valores en el área de Ciencias Naturales.

\section{Referencias bibliográficas.}

Aguilar, E. (2016). Investigación Científica. Quito, Ecuador: Ruiz.

Ayala, M., \& Ayala, S. (2018). Aprendizaje basado en problemas (APB) como técnica didáctica en extensión: construcción de conocimientos entre integrantes de Cooperativas Ellas Hacen en la Universidad Nacional de Formosa. (file://C:/Users/PC-146/Desktop/1065-5686-1-

PB\%20basado\%20en\%20problemas.pdf, Ed.) Rev. Fac. Agron. La Plata, 116(3), 12.

Burgo, O., León, J., Cáceres, M., Pérez, C., \& Espinoza, E. (2019). Algunas reflexiones sobre investigación e intervención educativa. Revista Cubana de Medicina Militar, 48(1), 15.

Chiva, I., \& Ramos, G. (Septiembre de 2015). Diseño y validación de una escala para evaluar las estrategias de gestión e intervención docente en educación primaria. Scielo. Redalyc. Revista Electrónica Actualidades Investigativas en Educación, 15(3), 1-24. 
García, M., \& Buitrago, Y. (2017). Modelo pedagógico de pensamiento complejo en diseño gráfico. Revista Logos, Ciencia \& Tecnología, 8(2), 117-131.

Gómez, R. (2012). Análisis de los métodos didácticos en la enseñanza. Universidad Nacional de Educación a Distancia (UNED), 32, 261-334.

Gómez, R. (21 de Octubre de 2016). Propuesta metodológica de enseñanza y aprendizaje para innovar la educación superior. Universidad de Costa Rica, 36(17), 12.

González, E. (2017 ). Factores que inciden en el rendimiento académico de los estudiantes de la Universidad Politécnica del Valle de Toluca. Revista Latinoamericana de Estudios Educativos, 47(1), 91-108.

Hernández, C., \& Guárate, A. (2017). Modelos didácticos: Para situaciones y contextos de aprendizaje (Vol. 146). Madrid, España: Narcea Ediciones.

Herrera, M., \& Zapata, P. (Junio de 2018). Estudio correlacional de estilos de aprendizaje de estudiantes con modalidad en ciencias naturales. Universidad Pedagógica Nacional(12), 17.

Herrera, V., Montes, I., Tapia, M., \& Barros, E. (2017). Diseño de un plan de mejoramiento como propuesta de intervención para el fortalecimiento de los procesos curriculares de una institución educativa. Barranquilla, Colombia: Universidad del Norte.

Iturralde, M., Bravo, B., \& Flores, A. (7 de Septiembre de 2017). Agenda actual en investigación en Didáctica de las Ciencias Naturales en América Latina y el Caribe. Revista Electrónica de Investigación Educativa, 19(3), 11.

López, M. (2015). Las estrategias didácticas en el proceso de enseñanza aprendizaje de los estudiantes de cuarto año de Educación General Básica en la Unidad Educativa "Manuela Espejo". Ambato, Ecuador: Universidad Técnológica Indoamérica.

López, N., \& Alvarado, D. (2017). Importancia del modelo pedagógico en la institución educativa Santa Catalina Labouré, Bolívar, Cauca. Popayan- Cauca : Universiad Católica de Manizales.

Mayoral, P. (2016). Estrategias didácticas para la enseñanza: el caso de un colegio en Colima, México. Colima, México: Instituto Tecnológico y de Estudios Superiores de Occidentes Obtenido de https://rei.iteso.mx/bitstream/handle/11117/3790/TOG\%20Pedro\%20Mayoral.p df?sequence $=3$

Muñoz, L., Montenegro, R., \& Aparicio, B. (8 de Septiembre de 2017). Uso de la Realidad Aumentada en la enseñanza-aprendizaje de ciencias naturales. In Memorias de Congresos UTP (pp. 96-101). 4to Congreso Internacional , 6. 
Necuzzi, C. (2018). Educación, enseñanza y didáctica en la contemporaneidad. Palermo , Buenos Aires: Cuadernos del Centro de Estudios en Diseño y Comunicación [Ensayos]. doi:ISSN 1668-0227

Ochoa, L. (2019). Recursos didácticos para el fortalecimiento del proceso de enseñanza aprendizaje en el área de Ciencias Naturales. Guayaquil, Ecuador: Universidad de Guayaquil: Facultad de Filosofía, Letras y Ciencias de la Educación.

Olivo, J. (Diciembre de 2017). Caracterización de estudiantes exitosos: Una aproximación al aprendizaje de las Ciencias Naturales. Revista de Investigación Educativa, 25, 114-143.

Orellana, C., Quintanilla, M., \& Páez, R. (2018). Concepciones sobre enseñanza y aprendizaje de las Ciencias Naturales de educadoras de párvulos en formación en Chile y sus relaciones con modelos de racionalidad científica. Pontificia Universidad Católica de Chile. Scielo, 24(4), 14.

Ortega, V., \& Gil, C. (11 de Noviembre de 2018). Estudio de aplicación de modelos didácticos de Ciencias Experimentales en un proyecto comunidad de aprendizaje. Universidad de Valladolid. ReiDoCrea, 8, 80, 9415.

Ortega, V., \& Puente, C. (2019). Estudio de aplicación de modelos didácticos de Ciencias Experimentales en un proyecto comunidad de aprendizaje. , 8. REiDoCrea: Revista Electrónica de Investigación y Docencia Creativa, 8, 80-94. 15.

Ortiz, J. (Abril de 2021). Mobile-learning como estrategia de refuerzo académico en el proceso de enseñanza aprendizaje de la matemática. Ceincia Digital , 17.

Patón, R., Fernández, J., \& Ferreiro, M. (10 de Abril de 2018). Aplicación de juegos didácticos como metodología de enseñanza. (file:///C:/Users/PC146/Downloads/Dialnet-

AplicacionDeJuegosDidacticosComoMetodologiaDeEnsen-6000065\%20(1).pdf, Ed.) Revista Digital de Educación Física, 18.

Pazmiño, J., Núñez, B., López, J., \& Pazmiño, M. (12 de Mayo de 2019). Inteligencia emocional: una competencia para fortalecer el desarrollo cognitivo, el pensamiento crítico, la toma de decisiones y el rendimiento académico . Ciencia Digital, doi:http://cienciadigital.org/revistacienciadigital2/index.php/CienciaDigital/artic le/view/472

Pérez, V. (2016). El lugar del examen en la Escuela: Estudio desde el campo de la Enseñanza de las Ciencias. Cantabria, España: Universidad de Cantabria.

Quintana, Y. (2018). Calidad educativa y gestión escolar: una relación dinámica. Educación y educadores. Universidad Industrial de Santander, 21(2), 259-281. 
Salazar, J. (2018). Evaluación de aprendizaje significativo y estilos de aprendizaje: Alcance, propuesta y desafíos en el aula. Tendencias pedagógicas. Universidad Autónoma de Madrid. Departamento de Didáctica y Teoría de la Educación, 16.

Sampieri, R., Fernández, C., \& Baptista, M. (2014). Metodología de la Investigación (Sexta edición ed.). (https://www.esup.edu.pe/descargas/dep_investigacion/Metodologia\%20de\%201 a\%20investigaci\%C3\%B3n\%205ta\%20Edici\%C3\%B3n.pdf, Ed.) Distrito Federal, México: McGRAW-HILL / INTERAMERICANA EDITORES, S.A. DE C.V.

Santana, M., \& Corral, K. (1 de Septiembre de 2020). Adaptación de recursos didácticos para la enseñanza en el área de ciencias naturales a niños con discapacidad visual de 7 mo grado de educación general básica en escuelas regulares de Manta. Revista Científica Multidisciplinaria SAPIENTIAE. ISSN: 2600-6030., 3(6), 1.

Tünnermann, C. (Marzo de 2011). Uso de la realidad aumentada como estrategia de aprendizaje para la enseñanza de las Ciencias Naturales. Unión de Universidades de América Latina y el Caribe, 48, 21-32.

Villanueva, S. (24 de Octubre de 2016). Alfa Cronbach, Prueba de regresión lineal, Prueba T, Chi cuadrada \& Coeficiente de correlación de Pearson. Pedagogía, 12.

Villavicencio, T. (2018). Gestión curricular en la aplicación de procesos pedagógicos y didácticos del área de matemática en la Institución Educativa Pública No 82295. Lima, Perú: Universidad Andina Simón Bolívar.

Villegas, C., Muñoz, F., \& Villegas, R. (Diciembre de 2009). Hábitos de estudio de los alumnos en el área de química orgánica y su impacto en el rendimiento académico. Universidad de Sonora. Investigación , 11, 11 . Obtenido de https://biotecnia.unison.mx/index.php/biotecnia/article/view/72/66

Zabalza, M. (2017). Didáctica de la educación infantil (Vol. 6). Madrid, España: Narcea Ediciones.

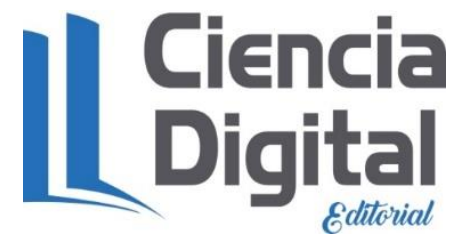


PARA CITAR EL ARTÍCULO INDEXADO.

Freire Pazmiño, J. C. (2021). Método didáctico para promover la calidad educativa en el aprendizaje de las ciencias naturales . AlfaPublicaciones, 3(3), 40-56. https://doi.org/10.33262/ap.v3i3.66

\section{Ciencia \\ LDigital}

El artículo que se publica es de exclusiva responsabilidad de los autores y no necesariamente reflejan el pensamiento de la Revista Alfa Publicaciones.

El artículo queda en propiedad de la revista y, por tanto, su publicación parcial y/o total en otro medio tiene que ser autorizado por el director de la Revista Alfa Publicaciones.
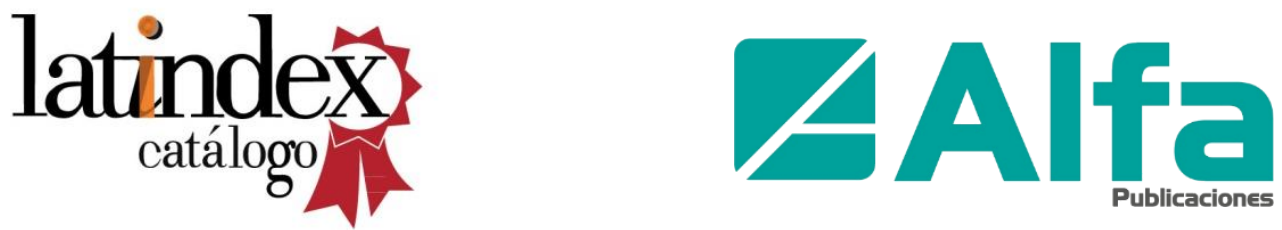